\title{
A QUASISTATIC CONTACT PROBLEM WITH ADHESION AND FRICTION FOR VISCOELASTIC MATERIALS
}

Abstract. We consider a mathematical model which describes the contact between a deformable body and a foundation. The contact is frictional and is modelled by a version of normal compliance condition and the associated Coulomb's law of dry friction in which adhesion of contact surfaces is taken into account. The evolution of the bonding field is described by a first order differential equation and the material's behaviour is modelled by a nonlinear viscoelastic constitutive law. We derive a variational formulation of the mechanical problem and prove the existence and uniqueness of a weak solution if the friction coefficient is sufficiently small. The proof is based on time-dependent variational inequalities, differential equations and the Banach fixed point theorem.

1. Introduction. Contact problems involving deformable bodies are quite frequent in industry as well as in daily life and play an important role in structural and mechanical systems. Because of the importance of these processes a considerable effort has been made in their modelling and numerical simulations. A first study of frictional contact problems within the framework of variational inequalities was made in [6]. The mathematical, mechanical and numerical state of the art can be found in [16]. The quasistatic contact problem with normal compliance and friction for viscoelastic materials was studied in [13].

In this paper we continue the study of this model in which moreover the adhesion of contact surfaces is taken into account. Models for dynamic or quasistatic process of frictionless adhesive contact between a deformable body and a foundation have been studied in [3, 4, 15, 16, 18, In [2] a model

2010 Mathematics Subject Classification: 47J20, 49J40, 74M10, 74M15.

Key words and phrases: viscoelastic materials, adhesion, normal compliance, fixed point, weak solution. 
of a contact problem with adhesion and friction was studied in which $\beta$ represents a continuous transition between total adhesive and pure frictional states. Also in [5] a quasistatic unilateral contact problem with local friction and adhesion for elastic materials was studied and an existence result for a friction coefficient small enough was established.

As in [8, 9], we use the bonding field as an additional state variable $\beta$, defined on the contact surface of the boundary. The variable is restricted to values $0 \leq \beta \leq 1$. When $\beta=0$ all the bonds are severed and there are no active bonds; when $\beta=1$ all the bonds are active; when $0<\beta<1$ it measures the fraction of active bonds and partial adhesion takes place. We refer the reader to the extensive bibliography on the subject in $[10,12,14-18]$. In this work we derive a variational formulation of the mechanical problem for which we prove the existence and uniqueness of a weak solution if the friction coefficient is sufficiently small, and obtain a partial regularity result for the solution.

The paper is structured as follows. In Section 2 we present some notations and give the variational formulation. In Section 3 we state and prove our main existence and uniqueness result, Theorem 2.1.

2. Problem statement and variational formulation. Let $\Omega \subset \mathbb{R}^{d}$ $(d=2,3)$ be a domain initially occupied by a viscoelastic body. $\Omega$ is supposed to be open, bounded, with a sufficiently regular boundary $\Gamma$ partitioned into three measurable parts, $\Gamma=\bar{\Gamma}_{1} \cup \bar{\Gamma}_{2} \cup \bar{\Gamma}_{3}$, where $\Gamma_{1}, \Gamma_{2}, \Gamma_{3}$ are disjoint open sets and meas $\Gamma_{1}>0$. The body is acted upon by a volume force of density $\varphi_{1}$ on $\Omega$ and a surface traction of density $\varphi_{2}$ on $\Gamma_{2}$. On $\Gamma_{3}$ the body is in adhesive and frictional contact with a foundation.

Thus, the classical formulation of the mechanical problem is as follows.

Problem $P_{1}$. Find a displacement field $u: \Omega \times[0, T] \rightarrow \mathbb{R}^{d}$ and a bonding field $\beta: \Gamma_{3} \times[0, T] \rightarrow[0,1]$ such that

$$
\begin{aligned}
& \operatorname{div} \sigma+\varphi_{1}=0 \quad \text { in } \Omega \times(0, T), \\
& \sigma=A \varepsilon(\dot{u})+B \varepsilon(u) \quad \text { in } \Omega \times(0, T), \\
& u=0 \quad \text { on } \Gamma_{1} \times(0, T), \\
& \sigma \nu=\varphi_{2} \quad \text { on } \Gamma_{2} \times(0, T), \\
& -\sigma_{\nu}=p\left(u_{\nu}\right)-c_{\nu} \beta^{2} R_{\nu}\left(u_{\nu}\right) \quad \text { on } \Gamma_{3} \times(0, T), \\
& \left\{\begin{array}{l}
\left|\sigma_{\tau}+c_{\tau} \beta^{2} R_{\tau}\left(u_{\tau}\right)\right| \leq \mu p\left(u_{\nu}\right) \\
\left|\sigma_{\tau}+c_{\tau} \beta^{2} R_{\tau}\left(u_{\tau}\right)\right|<\mu p\left(u_{\nu}\right) \Rightarrow \dot{u}_{\tau}=0 \quad \text { on } \Gamma_{3} \times(0, T), \\
\left|\sigma_{\tau}+c_{\tau} \beta^{2} R_{\tau}\left(u_{\tau}\right)\right|=\mu p\left(u_{\nu}\right) \Rightarrow \\
\exists \lambda \geq 0: \dot{u}_{\tau}=-\lambda\left(\sigma_{\tau}+c_{\tau} \beta^{2} R_{\tau}\left(u_{\tau}\right)\right),
\end{array}\right.
\end{aligned}
$$




$$
\begin{array}{ll}
\dot{\beta}=-\left[\beta\left(c_{\nu}\left|R_{\nu}\left(u_{\nu}\right)\right|^{2}+c_{\tau}\left|R_{\tau}\left(u_{\tau}\right)\right|^{2}\right)-\varepsilon_{a}\right]_{+} & \text {on } \Gamma_{3} \times(0, T), \\
u(0)=u_{0} & \text { in } \Omega, \\
\beta(0)=\beta_{0} & \text { on } \Gamma_{3} .
\end{array}
$$

We denote by $u$ the displacement field, by $\sigma$ the stress field and by $\varepsilon(u)$ the linearized strain tensor. Equation (2.1) is the equilibrium equation. Equation (2.2) represents the viscoelastic constitutive law of the material in which $A$ and $B$ are given nonlinear constitutive functions. Here and below, a dot above a variable represents a time derivative. We recall that in linear viscoelasticity the stress tensor $\sigma=\left(\sigma_{i j}\right)$ is given by

$$
\sigma_{i j}=a_{i j k h} \varepsilon_{k h}(\dot{u})+b_{i j k h} \varepsilon_{k h}(u),
$$

where $A=\left(a_{i j k h}\right)$ is the viscosity tensor and $B=\left(b_{i j k h}\right)$ is the elasticity tensor, for $i, j, k, h=1, \ldots, d$. (2.3) and (2.4) are the displacement and traction boundary conditions, respectively, in which $\nu$ denotes the unit outward normal vector on $\Gamma$, and $\sigma \nu$ represents the Cauchy stress vector. Condition (2.5) represents the normal compliance condition with adhesion and (2.6) is the associated Coulomb's law of dry friction on the contact surface $\Gamma_{3}$. Here $p$ is a given function, $\mu$ is the friction coefficient and the parameters $c_{\nu}, c_{\tau}$ and $\varepsilon_{a}$ are adhesion coefficients which may depend on $x \in \Gamma_{3}$. As in [18], $R_{\nu}, R_{\tau}$ are truncation operators defined by

$$
R_{\nu}(s)=\left\{\begin{array}{ll}
L & \text { if } s<-L, \\
-s & \text { if }-L \leq s \leq 0, \\
0 & \text { if } s>0,
\end{array} \quad R_{\tau}(v)= \begin{cases}v & \text { if }|v| \leq L \\
L v /|v| & \text { if }|v|>L\end{cases}\right.
$$

where $L>0$ is a characteristic length of the bonds. Equation (2.7) is an ordinary differential equation which describes the evolution of the bonding field and it was already used in [17]; here $[s]_{+}=\max (s, 0)$ for $s \in \mathbb{R}$. Since $\dot{\beta} \leq 0$ on $\Gamma_{3} \times(0, T)$, once debonding occurs, bonding cannot be reestablished. Also we wish to make it clear that from [11] it follows that the model does not allow for a complete debonding field in finite time. Finally, (2.8) and (2.9) represent respectively the initial displacement field and the initial bonding field. We recall that the inner products and the corresponding norms on $\mathbb{R}^{d}$ and $S_{d}$ are given by

$$
\begin{aligned}
& u . v=u_{i} v_{i}, \quad|v|=(v \cdot v)^{1 / 2} \quad \forall u, v \in \mathbb{R}^{d}, \\
& \sigma . \tau=\sigma_{i j} \tau_{i j}, \quad|\tau|=(\tau . \tau)^{1 / 2} \quad \forall \sigma, \tau \in S_{d},
\end{aligned}
$$

where $S_{d}$ is the space of second order symmetric tensors on $\mathbb{R}^{d}(d=2,3)$. Here and below, the indices $i$ and $j$ run between 1 and $d$ and the summation convention over repeated indices is adopted. 
Now, to proceed with the variational formulation, we need the following function spaces:

$$
\begin{aligned}
& H=\left(L^{2}(\Omega)\right)^{d}, \quad H_{1}=\left(H^{1}(\Omega)\right)^{d}, \\
& Q=\left\{\tau=\left(\tau_{i j}\right) ; \tau_{i j}=\tau_{j i} \in L^{2}(\Omega)\right\}, \quad Q_{1}=\{\sigma \in Q ; \operatorname{div} \sigma \in H\} .
\end{aligned}
$$

Note that $H$ and $Q$ are real Hilbert spaces endowed with the respective canonical inner products

$$
\langle u, v\rangle_{H}=\int_{\Omega} u_{i} v_{i} d x, \quad\langle\sigma, \tau\rangle_{Q}=\int_{\Omega} \sigma_{i j} \tau_{i j} d x .
$$

The linearized strain tensor is

$$
\varepsilon(u)=\left(\varepsilon_{i j}(u)\right)=\frac{1}{2}\left(u_{i, j}+u_{j, i}\right) ;
$$

$\operatorname{div} \sigma=\left(\sigma_{i j, j}\right)$ is the divergence of $\sigma$. For every element $v \in H_{1}$ we denote by $v_{\nu}$ and $v_{\tau}$ the normal and tangential components of $v$ on the boundary $\Gamma$, given by

$$
v_{\nu}=v \cdot \nu, \quad v_{\tau}=v-v_{\nu} \nu .
$$

Similarly, for a regular function $\sigma \in Q_{1}$, we define its normal and tangential components by

$$
\sigma_{\nu}=(\sigma \nu) . \nu, \quad \sigma_{\tau}=\sigma \nu-\sigma_{\nu} \nu
$$

and we recall that the following Green's formula holds:

$$
\langle\sigma, \varepsilon(v)\rangle_{Q}+\langle\operatorname{div} \sigma, v\rangle_{H}=\int_{\Gamma} \sigma \nu \cdot v d a \quad \forall v \in H_{1},
$$

where $d a$ is the surface measure element. Let $V$ be the closed subspace of $H_{1}$ defined by

$$
V=\left\{v \in H_{1} ; v=0 \text { on } \Gamma_{1}\right\} .
$$

Since meas $\Gamma_{1}>0$, the following Korn's inequality holds [6]:

$$
\|\varepsilon(v)\|_{Q} \geq c_{\Omega}\|v\|_{H_{1}} \quad \forall v \in V,
$$

where the constant $c_{\Omega}>0$ depends only on $\Omega$ and $\Gamma_{1}$. We equip $V$ with the inner product

$$
(u, v)_{V}=\langle\varepsilon(u), \varepsilon(v)\rangle_{Q}
$$

and $\|\cdot\|_{V}$ is the associated norm. It follows from Korn's inequality (2.9) that the norms $\|\cdot\|_{H_{1}}$ and $\|\cdot\|_{V}$ are equivalent on $V$. Then $\left(V,\|\cdot\|_{V}\right)$ is a real Hilbert space. Moreover by Sobolev's trace theorem, there exists $d_{\Omega}>0$ which depends only on the domain $\Omega, \Gamma_{1}$ and $\Gamma_{3}$ such that

$$
\|v\|_{\left(L^{2}\left(\Gamma_{3}\right)\right)^{d}} \leq d_{\Omega}\|v\|_{V} \quad \forall v \in V .
$$

For $p \in[1, \infty]$, we use the standard norm of $L^{p}(0, T ; V)$. We also use the Sobolev space $W^{1, \infty}(0, T ; V)$ equipped with the norm

$$
\|v\|_{W^{1, \infty}(0, T ; V)}=\|v\|_{L^{\infty}(0, T ; V)}+\|\dot{v}\|_{L^{\infty}(0, T ; V)} .
$$


For every real Banach space $\left(X,\|\cdot\|_{X}\right)$ and $T>0$ we use the notation $C([0, T] ; X)$ for the space of continuous functions from $[0, T]$ to $X$; recall that $C([0, T] ; X)$ is a real Banach space with the norm

$$
\|x\|_{C([0, T] ; X)}=\max _{t \in[0, T]}\|x(t)\|_{X} .
$$

We suppose that the body forces and surface tractions have the regularity

$$
\varphi_{1} \in C([0, T] ; H), \quad \varphi_{2} \in C\left([0, T] ;\left(L^{2}\left(\Gamma_{2}\right)\right)^{d}\right),
$$

and we denote by $f(t)$ the element of $V$ defined by

$$
(f(t), v)_{V}=\int_{\Omega} \varphi_{1}(t) \cdot v d x+\int_{\Gamma_{2}} \varphi_{2}(t) \cdot v d a \quad \forall v \in V, t \in[0, T] .
$$

Using (2.11) and (2.12) we obtain

$$
f \in C([0, T] ; V) \text {. }
$$

Also we define the functional $j: V \times V \rightarrow \mathbb{R}$ by

$$
j(v, w)=\int_{\Gamma_{3}} \mu p\left(v_{\nu}\right)\left|w_{\tau}\right| d a \quad \forall v, w \in V,
$$

where the normal compliance $p$ is assumed to satisfy

$$
\left\{\begin{array}{l}
\text { (a) } p: \Gamma_{3} \times \mathbb{R} \rightarrow \mathbb{R}_{+} ; \\
\text {(b) there exists } L_{p}>0 \text { such that }\left|p\left(x, r_{1}\right)-p\left(x, r_{2}\right)\right| \leq L_{p}\left|r_{1}-r_{2}\right| \\
\quad \text { for all } r_{1}, r_{2} \in \mathbb{R} \text { and a.e. } x \in \Gamma_{3} ; \\
\text { (c) } x \mapsto p(x, r) \text { is measurable on } \Gamma_{3} \text { for any } r \in \mathbb{R} ; \\
\text { (d) } p(x, r)=0 \text { for all } r \leq 0 \text { and a.e. } x \in \Gamma_{3} .
\end{array}\right.
$$

$\mu$ is the friction coefficient and we assume that

$$
\mu \in L^{\infty}\left(\Gamma_{3}\right) \text { and } \mu \geq 0 \text { a.e. on } \Gamma_{3} .
$$

In the study of problem $P_{1}$ we assume that the viscosity operator $A$ satisfies

$$
\left\{\begin{array}{l}
\text { (a) } A: \Omega \times S_{d} \rightarrow S_{d} ; \\
\text { (b) there exists } M_{A}>0 \text { such that } \\
\quad\left|A\left(x, \varepsilon_{1}\right)-A\left(x, \varepsilon_{2}\right)\right| \leq M_{A}\left|\varepsilon_{1}-\varepsilon_{2}\right| \\
\quad \text { for all } \varepsilon_{1}, \varepsilon_{2} \text { in } S_{d} \text { and a.e. } x \text { in } \Omega ; \\
\text { (c) there exists } m_{A}>0 \text { such that } \\
\quad\left(A\left(x, \varepsilon_{1}\right)-A\left(x, \varepsilon_{2}\right)\right) \cdot\left(\varepsilon_{1}-\varepsilon_{2}\right) \geq m_{A}\left|\varepsilon_{1}-\varepsilon_{2}\right|^{2} \\
\quad \text { for all } \varepsilon_{1}, \varepsilon_{2} \text { in } S_{d} \text { and a.e. } x \text { in } \Omega ; \\
\text { (d) } x \mapsto A(x, \varepsilon) \text { is Lebesgue measurable on } \Omega \\
\\
\quad \text { for any } \varepsilon \text { in } S_{d} ; \\
\text { (e) } x \mapsto A(x, 0) \in Q .
\end{array}\right.
$$


The elasticity operator $B$ satisfies

$$
\left\{\begin{array}{l}
\text { (a) } B: \Omega \times S_{d} \rightarrow S_{d} \\
\text { (b) there exists } M_{B}>0 \text { such that } \\
\quad\left|B\left(x, \varepsilon_{1}\right)-B\left(x, \varepsilon_{2}\right)\right| \leq M_{B}\left|\varepsilon_{1}-\varepsilon_{2}\right|, \\
\quad \text { for all } \varepsilon_{1}, \varepsilon_{2} \text { in } S_{d} \text { and a.e. } x \text { in } \Omega ; \\
\text { (c) } x \mapsto B(x, \varepsilon) \text { is Lebesgue measurable on } \Omega \\
\quad \text { for any } \varepsilon \text { in } S_{d} ; \\
\text { (e) } x \mapsto B(x, 0) \in Q .
\end{array}\right.
$$

As in [18] we suppose that the adhesion coefficients $c_{\nu}, c_{\tau}$ and $\varepsilon_{a}$ satisfy

$$
c_{\nu}, c_{\tau} \in L^{\infty}\left(\Gamma_{3}\right), \quad \varepsilon_{a} \in L^{\infty}\left(\Gamma_{3}\right), \quad c_{\nu}, c_{\tau}, \varepsilon_{a} \geq 0 \quad \text { a.e. on } \Gamma_{3} .
$$

We assume that the initial data satisfy

$$
\begin{gathered}
u_{0} \in V, \\
\beta_{0} \in L^{2}\left(\Gamma_{3}\right), \quad 0 \leq \beta_{0} \leq 1 \quad \text { a.e. on } \Gamma_{3} .
\end{gathered}
$$

Next, we define the functional $r: L^{2}\left(\Gamma_{3}\right) \times V \times V \rightarrow \mathbb{R}$ by

$$
r(\beta, u, v)=\int_{\Gamma_{3}}\left(p\left(u_{\nu}\right) v_{\nu}-c_{\nu} \beta^{2} R_{\nu}\left(u_{\nu}\right) v_{\nu}+c_{\tau} \beta^{2} R_{\tau}\left(u_{\tau}\right) \cdot v_{\tau}\right) d a .
$$

Finally, we need the following set for the bonding fields:

$$
\mathcal{O}=\left\{\theta:[0, T] \rightarrow L^{2}\left(\Gamma_{3}\right) ; 0 \leq \theta(t) \leq 1 \forall t \in[0, T] \text {, a.e. on } \Gamma_{3}\right\} \text {. }
$$

Now, assuming the solution to be sufficiently regular, and using Green's formula, we see that Problem $P_{1}$ has the following variational formulation.

Problem $P_{2}$. Find a displacement field $u \in C^{1}([0, T] ; V)$ and a bonding field $\beta \in W^{1, \infty}\left(0, T ; L^{2}\left(\Gamma_{3}\right)\right) \cap \mathcal{O}$ such that

$$
\begin{aligned}
& \langle A \varepsilon(\dot{u}(t)), \varepsilon(v)-\varepsilon(\dot{u}(t))\rangle_{Q}+\langle B \varepsilon(u(t)), \varepsilon(v)-\varepsilon(\dot{u}(t))\rangle_{Q} \\
& +j(u(t), v)-j(u(t), \dot{u}(t))+r(\beta(t), u(t), v-\dot{u}(t)) \\
& \geq(f(t), v-\dot{u}(t))_{V} \quad \forall v \in V, t \in[0, T], \\
& (2.21) \quad \dot{\beta}(t)=-\left[\beta ( t ) \left(c_{\nu}\left|R_{\nu}\left(u_{\nu}(t)\right)\right|^{2}\right.\right. \\
& \left.\left.+c_{\tau}\left|R_{\tau}\left(u_{\tau}(t)\right)\right|^{2}\right)-\varepsilon_{a}\right]_{+} \quad \text { a.e. } t \in(0, T), \\
& \text { (2.23) } \quad \beta(0)=\beta_{0} \quad \text { on } \Gamma_{3} \text {. }
\end{aligned}
$$$$
\text { (2.22) } u(0)=u_{0} \quad \text { in } \Omega \text {, }
$$

Our main result, which will be established in the next section, is the following theorem. 
TheOREM 2.1. Let $T>0$ and assume that (2.11) and (2.13)-(2.19) hold. Then there exists a constant $\mu_{0}>0$ such that if

$$
\|\mu\|_{L^{\infty}\left(\Gamma_{3}\right)}<\mu_{0}
$$

then Problem $P_{2}$ has a unique solution.

3. Existence and uniqueness result. The proof of Theorem 2.1 will be carried out in several steps. In the first step, for given $\eta \in C([0, T] ; V)$ and $g \in C([0, T] ; V)$ we consider the following variational problem.

Problem $P_{\eta g}$. Find $v_{\eta g}:[0, T] \rightarrow V$ such that

$$
\begin{aligned}
& \left\langle A \varepsilon\left(v_{\eta g}(t)\right), \varepsilon(w)-\varepsilon\left(v_{\eta g}(t)\right)\right\rangle_{Q}+\left(\eta(t), w-v_{\eta g}(t)\right)_{V}+j(g(t), w) \\
& -j\left(g(t), v_{\eta g}(t)\right) \geq\left(f(t), w-v_{\eta g}(t)\right)_{V} \quad \forall w \in V, t \in[0, T] .
\end{aligned}
$$

We show the following result.

Lemma 3.1. Problem $P_{\eta g}$ has a unique solution and the solution satisfies $v_{\eta g} \in C([0, T] ; V)$.

Proof. We define the operator $C: V \rightarrow V$ by

$$
(C v, w)_{V}=\langle A \varepsilon(v), \varepsilon(w)\rangle_{Q} \quad \forall v, w \in V .
$$

It follows from assumption (2.15) that $C$ is a strongly monotone and Lipschitz continuous operator. Next, let $t \in[0, T]$. The functional $j(g(t), \cdot)$ is a continuous seminorm on $V$, so by a classical argument of elliptic variational inequalities [1], there exists a unique element $v_{\eta g}(t) \in V$ such that

$$
\begin{aligned}
\left\langle A \varepsilon\left(v_{\eta g}(t)\right), \varepsilon(w)-\varepsilon\left(v_{\eta g}(t)\right)\right\rangle_{Q}+j(g(t), w)-j\left(g(t), v_{\eta g}(t)\right) & \\
& \geq\left(f(t)-\eta(t), w-v_{\eta g}(t)\right)_{V} \quad \forall w \in V .
\end{aligned}
$$

Thus, we use (3.2) to see that $v_{\eta g}(t)$ is the unique element which solves (3.1), for each $t \in[0, T]$. Now, let $t_{1}, t_{2} \in[0, T]$. We write (3.2) for $t=t_{1}$ and $w=v_{\eta g}\left(t_{2}\right)$. Then for $t=t_{2}$ and $w=v_{\eta g}\left(t_{1}\right)$, by adding the resulting inequalities we obtain

$$
\begin{aligned}
& \left\langle A \varepsilon\left(v_{\eta g}\left(t_{1}\right)\right)-A \varepsilon\left(v_{\eta g}\left(t_{2}\right)\right), \varepsilon\left(v_{\eta g}\left(t_{1}\right)\right)-\varepsilon\left(v_{\eta g}\left(t_{2}\right)\right)\right\rangle_{Q} \\
& \leq\left(f\left(t_{1}\right)-f\left(t_{2}\right), v_{\eta g}\left(t_{1}\right)-v_{\eta g}\left(t_{2}\right)\right)_{V}-\left(\eta\left(t_{1}\right)-\eta\left(t_{2}\right), v_{\eta g}\left(t_{1}\right)-v_{\eta g}\left(t_{2}\right)\right)_{V} \\
& \quad+j\left(g\left(t_{1}\right), v_{\eta g}\left(t_{2}\right)\right)-j\left(g\left(t_{1}\right), v_{\eta g}\left(t_{1}\right)\right)+j\left(g\left(t_{2}\right), v_{\eta g}\left(t_{1}\right)\right)-j\left(g\left(t_{2}\right), v_{\eta g}\left(t_{2}\right)\right) .
\end{aligned}
$$

Using (2.15)(c), (2.13)(b), (2.17), and (2.10), we see that

$$
\begin{aligned}
m_{A}\left\|v_{\eta g}\left(t_{1}\right)-v_{\eta g}\left(t_{2}\right)\right\|_{V}^{2} \leq & d_{\Omega}^{2}\|\mu\|_{L^{\infty}\left(\Gamma_{3}\right)}\left\|v_{\eta g}\left(t_{1}\right)-v_{\eta g}\left(t_{2}\right)\right\|_{V}^{2} \\
& +\left\|\eta\left(t_{1}\right)-\eta\left(t_{2}\right)\right\|_{V}\left\|v_{\eta g}\left(t_{1}\right)-v_{\eta g}\left(t_{2}\right)\right\|_{V} \\
& +d_{\Omega}^{2}\left\|g\left(t_{1}\right)-g\left(t_{2}\right)\right\|_{V}\left\|v_{\eta g}\left(t_{1}\right)-v_{\eta g}\left(t_{2}\right)\right\|_{V} \\
& +\left\|f\left(t_{1}\right)-f\left(t_{2}\right)\right\|_{V}\left\|v_{\eta g}\left(t_{1}\right)-v_{\eta g}\left(t_{2}\right)\right\|_{V} .
\end{aligned}
$$


Then, if we take

$$
\mu_{0}=m_{A} / d_{\Omega}^{2},
$$

it follows that if $\|\mu\|_{L^{\infty}\left(\Gamma_{3}\right)}<\mu_{0}$, there exists a constant $c_{1}>0$ such that

$$
\begin{aligned}
& \left\|v_{\eta g}\left(t_{1}\right)-v_{\eta g}\left(t_{2}\right)\right\|_{V} \\
& \quad \leq c_{1}\left(\left\|f\left(t_{1}\right)-f\left(t_{2}\right)\right\|_{V}+\left\|g\left(t_{1}\right)-g\left(t_{2}\right)\right\|_{V}+\left\|\eta\left(t_{1}\right)-\eta\left(t_{2}\right)\right\|_{V}\right) .
\end{aligned}
$$

As $f \in C([0, T] ; V), g \in C([0, T] ; V)$ and $\eta \in C([0, T] ; V)$, we deduce that $v_{\eta g} \in C([0, T] ; V)$.

Now, let us consider the operator $\Lambda_{\eta}: C([0, T] ; V) \rightarrow C([0, T] ; V)$ defined by

$$
\Lambda_{\eta} g=g_{\eta}, \quad g \in C([0, T] ; V)
$$

where

$$
g_{\eta}(t)=u_{0}+\int_{0}^{t} v_{\eta g}(s) d s \quad \text { for } t \in[0, T] .
$$

We have

Lemma 3.2. The operator $\Lambda_{\eta}$ has a unique fixed point $g_{\eta}^{*} \in C([0, T] ; V)$.

Proof. We refer the reader to [13, Proposition 4.2].

Next, for $\eta \in C([0, T] ; V)$, we denote by $g_{\eta}^{*}$ the fixed point given in Lemma 3.2. Let $v_{\eta} \in C([0, T] ; V)$ be the function defined by

$$
v_{\eta}=v_{\eta g_{\eta}^{*}}
$$

Using (3.3) and (3.4), let $u_{\eta}:[0, T] \rightarrow V$ be the function

$$
u_{\eta}(t)=g_{\eta}^{*}(t)=u_{0}+\int_{0}^{t} v_{\eta}(s) d s \quad \text { for } t \in[0, T] .
$$

Now, we consider the following problem.

Problem $P_{\eta \beta}$. Find a bonding field $\beta_{\eta}:[0, T] \rightarrow L^{2}\left(\Gamma_{3}\right)$ such that

$$
\begin{aligned}
& \dot{\beta}_{\eta}(t)=-\left[\beta _ { \eta } ( t ) \left(c_{\nu}\left|R_{\nu}\left(u_{\eta \nu}(t)\right)\right|^{2}\right.\right. \\
& \left.\left.+c_{\tau}\left|R_{\tau}\left(u_{\eta \tau}(t)\right)\right|^{2}\right)-\varepsilon_{a}\right]_{+} \quad \text { a.e. } t \in(0, T), \\
& \beta_{\eta}(0)=\beta_{0} \quad \text { on } \Gamma_{3} .
\end{aligned}
$$

We have the following result.

Lemma 3.3. There exists a unique solution to Problem $P_{\eta \beta}$ and it satisfies

$$
\beta_{\eta} \in W^{1, \infty}\left(0, T ; L^{2}\left(\Gamma_{3}\right)\right) \cap \mathcal{O} .
$$

Proof. Let $k>0$ and let

$$
X=\left\{\beta \in C\left([0, T] ; L^{2}\left(\Gamma_{3}\right)\right) ; \sup _{t \in[0, T]}\left[\exp (-k t)\|\beta(t)\|_{L^{2}\left(\Gamma_{3}\right)}\right]<+\infty\right\} .
$$


$X$ is a Banach space with the norm

$$
\|\beta\|_{X}=\sup _{t \in[0, T]}\left[\exp (-k t)\|\beta(t)\|_{L^{2}\left(\Gamma_{3}\right)}\right],
$$

which is equivalent to the standard norm $\|\cdot\|_{C\left([0, T] ; L^{2}\left(\Gamma_{3}\right)\right)}$. Consider the mapping $T: X \rightarrow X$ defined by

$$
T \beta(t)=\beta_{0}-\int_{0}^{t}\left[\beta(s)\left(c_{\nu}\left|R_{\nu}\left(u_{\eta \nu}(s)\right)\right|^{2}+c_{\tau}\left|R_{\tau}\left(u_{\eta \tau}(s)\right)\right|^{2}\right)-\varepsilon_{a}\right]_{+} d a .
$$

Using that $\left|R_{r}\left(u_{\eta r}\right)\right| \leq L, r=\nu, \tau$, it follows that there exists a constant $c_{2}>0$ such that

$$
\left\|T \beta_{1}(t)-T \beta_{2}(t)\right\|_{L^{2}\left(\Gamma_{3}\right)} \leq c_{2} \int_{0}^{t}\left\|\beta_{1}(s)-\beta_{2}(s)\right\|_{L^{2}\left(\Gamma_{3}\right)} d s .
$$

Since

$$
\begin{aligned}
\int_{0}^{t}\left\|\beta_{1}(s)-\beta_{2}(s)\right\|_{L^{2}\left(\Gamma_{3}\right)} d s & =\int_{0}^{t} e^{k s}\left(e^{-k s}\left\|\beta_{1}(s)-\beta_{2}(s)\right\|_{L^{2}\left(\Gamma_{3}\right)}\right) d s \\
& \leq\left\|\beta_{1}-\beta_{2}\right\|_{X_{1}} \frac{e^{k t}}{k},
\end{aligned}
$$

this inequality implies that

$$
\left\|T \beta_{1}-T \beta_{2}\right\|_{X} \leq \frac{c_{2}}{k}\left\|\beta_{1}-\beta_{2}\right\|_{X} .
$$

The inequality (3.9) shows that for $k>c_{2}, T$ is a contraction. Thus, by the Banach fixed point theorem, $T$ has a unique fixed point $\beta_{\eta}$ which satisfies (3.7) and (3.8). The regularity $\beta_{\eta} \in \mathcal{O}$ is a consequence of (3.8) and (2.19); see [18] for details.

Moreover, using the Riesz representation theorem we define the function $\Lambda:[0, T] \rightarrow V$ by

$$
\begin{aligned}
(\Lambda \eta(t), w)_{V}=\left\langle B \varepsilon\left(u_{\eta}(t)\right), \varepsilon(w)\right\rangle_{Q}+r\left(\beta_{\eta}(t),\right. & \left.u_{\eta}(t), w\right), \\
& \forall w \in V, t \in[0, T] .
\end{aligned}
$$

Lemma 3.3. For each $\eta \in C([0, T] ; V)$ the function $\Lambda \eta:[0, T] \rightarrow V$ belongs to $C([0, T] ; V)$. Moreover, there exists a unique $\eta^{*} \in C([0, T] ; V)$ such that $\Lambda \eta^{*}=\eta^{*}$.

Proof. Let $\eta \in C([0, T] ; V)$ and $t_{1}, t_{2} \in[0, T]$. Using (3.10), it follows that there exists a constant $C_{1}>0$ such that

$$
\begin{aligned}
\left\|\Lambda \eta\left(t_{1}\right)-\Lambda \eta\left(t_{2}\right)\right\|_{V} \leq & \left\|B \varepsilon\left(u_{\eta}\left(t_{1}\right)\right)-B \varepsilon\left(u_{\eta}\left(t_{2}\right)\right)\right\|_{Q} \\
& +C_{1}\left(\left\|\beta_{\eta}^{2}\left(t_{1}\right) R_{\tau}\left(u_{\eta \tau}\left(t_{1}\right)\right)-\beta_{\eta}^{2}\left(t_{2}\right) R_{\tau}\left(u_{\eta \tau}\left(t_{2}\right)\right)\right\|_{L^{2}\left(\Gamma_{3}\right)}\right. \\
& \left.+\left\|p\left(u_{\eta \nu}\left(t_{1}\right)\right)-p\left(u_{\eta \nu}\left(t_{2}\right)\right)\right\|_{L^{2}\left(\Gamma_{3}\right)}\right) \\
& +C_{1}\left\|\beta_{\eta}^{2}\left(t_{1}\right) R_{\nu}\left(u_{\eta \nu}\left(t_{1}\right)\right)-\beta_{\eta}^{2}\left(t_{2}\right) R_{\nu}\left(u_{\eta \nu}\left(t_{2}\right)\right)\right\|_{L^{2}\left(\Gamma_{3}\right)} .
\end{aligned}
$$


Using the properties (see [16]) of the operators $R_{\nu}, R_{\tau}$ such that

$$
\begin{aligned}
& \left|R_{r}\left(u_{\eta r}\right)\right| \leq L, \quad r=\nu, \tau, \quad\left|R_{\nu}(a)-R_{\nu}(b)\right| \leq|a-b| \quad \forall a, b \in \mathbb{R}, \\
& \left|R_{\tau}(a)-R_{\tau}(b)\right| \leq|a-b| \quad \forall a, b \in \mathbb{R}^{d},
\end{aligned}
$$

(2.13)(b), (2.16), and that $0 \leq \beta_{\eta}(t) \leq 1$ for all $t \in[0, T]$, it follows that there exists a constant $C_{2}>0$ such that

$$
\begin{aligned}
\| \Lambda \eta\left(t_{1}\right)- & \Lambda \eta\left(t_{2}\right) \|_{V} \\
& \leq C_{2}\left(\left\|u_{\eta}\left(t_{1}\right)-u_{\eta}\left(t_{2}\right)\right\|_{V}+\left\|\beta_{\eta}\left(t_{1}\right)-\beta_{\eta}\left(t_{2}\right)\right\|_{L^{2}\left(\Gamma_{3}\right)}\right) .
\end{aligned}
$$

Since $u_{\eta} \in C^{1}([0, T] ; V)$ and $\beta_{\eta} \in W^{1, \infty}(0, T ; V)$ we deduce from inequality (3.11) that $\Lambda \eta \in C([0, T] ; V)$.

Let now $\eta_{1}, \eta_{2} \in C([0, T] ; V)$. For $t \in[0, T]$ we integrate (3.7) with the initial condition (3.8) to obtain

$$
\beta_{\eta_{i}}(t)=\beta_{0}-\int_{0}^{t}\left[\beta_{\eta_{i}}(s)\left(c_{\nu}\left|R_{\nu}\left(u_{\eta_{i} \nu}(s)\right)\right|^{2}+c_{\tau}\left|R_{\tau}\left(u_{\eta_{i} \tau}(s)\right)\right|^{2}\right)-\varepsilon_{a}\right]_{+} d a .
$$

Hence there exists a constant $C_{3}>0$ such that

$$
\begin{aligned}
& \left\|\beta_{\eta_{1}}(t)-\beta_{\eta_{2}}(t)\right\|_{L^{2}\left(\Gamma_{3}\right)} \\
& \leq C_{3} \int_{0}^{t}\left\|\beta_{\eta_{1}}(s)\left|R_{\nu}\left(u_{\eta_{1} \nu}(s)\right)\right|^{2}-\beta_{\eta_{2}}(s)\left|R_{\nu}\left(u_{\eta_{2} \nu}(s)\right)\right|^{2}\right\|_{L^{2}\left(\Gamma_{3}\right)} d s \\
& \quad+C_{3} \int_{0}^{t}\left\|\beta_{\eta_{1}}(s)\left|R_{\tau}\left(u_{\eta_{1} \tau}(s)\right)\right|^{2}-\beta_{\eta_{2}}(s)\left|R_{\tau}\left(u_{\eta_{2} \tau}(s)\right)\right|^{2}\right\|_{L^{2}\left(\Gamma_{3}\right)} d s .
\end{aligned}
$$

We use the definition of the truncation operators $R_{\nu}, R_{\tau}$ and write

$$
\beta_{\eta_{1}}(s)=\beta_{\eta_{1}}(s)-\beta_{\eta_{2}}(s)+\beta_{\eta_{2}}(s) .
$$

After some elementary calculations we find that there exists a constant $C_{4}>0$ such that

$$
\begin{aligned}
& \left\|\beta_{\eta_{1}}(t)-\beta_{\eta_{2}}(t)\right\|_{L^{2}\left(\Gamma_{3}\right)} \leq C_{4} \int_{0}^{t}\left\|\beta_{\eta_{1}}(s)-\beta_{\eta_{2}}(s)\right\|_{L^{2}\left(\Gamma_{3}\right)} d s \\
& \quad+C_{4} \int_{0}^{t}\left\|u_{\eta_{1} \nu}(s)-u_{\eta_{2} \nu}(s)\right\|_{L^{2}\left(\Gamma_{3}\right)} d s+C_{4} \int_{0}^{t}\left\|u_{\eta_{1} \tau}(s)-u_{\eta_{2} \tau}(s)\right\|_{\left(L^{2}\left(\Gamma_{3}\right)\right)^{d}} d s .
\end{aligned}
$$

Using (2.10), it follows that

$$
\begin{aligned}
\left\|\beta_{\eta_{1}}(t)-\beta_{\eta_{2}}(t)\right\|_{L^{2}\left(\Gamma_{3}\right)} \leq & C_{4} \int_{0}^{t}\left\|\beta_{\eta_{1}}(s)-\beta_{\eta_{2}}(s)\right\|_{L^{2}\left(\Gamma_{3}\right)} d s \\
& +2 C_{4} d_{\Omega} \int_{0}^{t}\left\|u_{\eta_{1}}(s)-u_{\eta_{2}}(s)\right\|_{V} d s .
\end{aligned}
$$


Using a Gronwall-type inequality, we deduce that there exists a constant $C_{5}>0$ such that

$$
\left\|\beta_{\eta_{1}}(t)-\beta_{\eta_{2}}(t)\right\|_{L^{2}\left(\Gamma_{3}\right)} \leq C_{5} \int_{0}^{t}\left\|u_{\eta_{1}}(s)-u_{\eta_{2}}(s)\right\|_{V} d s .
$$

On the other hand, using arguments similar to those in the proof of (3.12), we find that there exists a constant $C_{6}>0$ such that

$$
\left\|\Lambda \eta_{1}(t)-\Lambda \eta_{2}(t)\right\|_{V} \leq C_{6}\left(\left\|u_{\eta_{1}}(t)-u_{\eta_{2}}(t)\right\|_{V}+\left\|\beta_{\eta_{1}}(t)-\beta_{\eta_{2}}(t)\right\|_{L^{2}\left(\Gamma_{3}\right)}\right) .
$$

Hence, by (3.12) we have

$$
\begin{aligned}
& \left\|\Lambda \eta_{1}(t)-\Lambda \eta_{2}(t)\right\|_{V} \\
& \quad \leq C_{6}\left\|u_{\eta_{1}}(t)-u_{\eta_{2}}(t)\right\|_{V}+C_{5} C_{6} \int_{0}^{t}\left\|u_{\eta_{1}}(s)-u_{\eta_{2}}(s)\right\|_{V} d s .
\end{aligned}
$$

On the other hand, the function $u_{\eta_{i}}$ satisfies the inequality

$$
\begin{aligned}
\left\langle A \varepsilon\left(v_{\eta i}(t)\right), \varepsilon(w\right. & \left.\left.-v_{\eta i}(t)\right)\right\rangle_{Q}+\left(\eta_{i}(t), w-v_{\eta i}(t)\right)_{V}+j\left(u_{\eta i}(t), w\right) \\
& -j\left(u_{\eta i}(t), v_{\eta i}(t)\right) \geq\left(f(t), w-v_{\eta i}(t)\right)_{V} \quad \forall w \in V,
\end{aligned}
$$

where $i=1,2$ and $t \in[0, T]$. It follows from (3.14) and the estimate in the proof of Lemma 3.1 that there exists a constant $C>0$ such that

$$
\begin{aligned}
& \left\|u_{\eta_{1}}(t)-u_{\eta_{2}}(t)\right\|_{V} \leq \int_{0}^{t}\left\|v_{\eta_{1}}(s)-v_{\eta_{2}}(s)\right\|_{V} d s \\
& \quad \leq C \int_{0}^{t}\left\|\eta_{1}(s)-\eta_{2}(s)\right\|_{V} d s+C \int_{0}^{t}\left\|u_{\eta_{1}}(s)-u_{\eta_{2}}(s)\right\|_{V} d s \quad \text { for } t \in[0, T] .
\end{aligned}
$$

Using now a Gronwall-type inequality we get

$$
\left\|u_{\eta_{1}}(t)-u_{\eta_{2}}(t)\right\|_{V} \leq C \int_{0}^{t}\left\|\eta_{1}(s)-\eta_{2}(s)\right\|_{V} d s \quad \forall t \in[0, T] .
$$

From (3.13) and (3.15) it follows that there exists a constant $C^{\prime}>0$ such that

$$
\left\|\Lambda \eta_{1}(t)-\Lambda \eta_{2}(t)\right\|_{V} \leq C^{\prime} \int_{0}^{t}\left\|\eta_{1}(s)-\eta_{2}(s)\right\|_{V} d s
$$

Let now $k>0$, and denote

$$
\|\eta\|_{k}=\sup _{t \in[0, T]}\left[\exp (-k t)\|\eta(t)\|_{V}\right] \quad \forall \eta \in C([0, T] ; V) .
$$

Clearly $\|\cdot\|_{k}$ defines a norm on the space $C([0, T] ; V)$ which is equivalent to the standard norm $\|\cdot\|_{C([0, T] ; V)}$. Using (3.16) and arguments similar to those in the proof of (3.9), after some calculations we find that there exists 
a constant $c>0$ such that

$$
\left\|\Lambda \eta_{1}-\Lambda \eta_{2}\right\|_{k} \leq \frac{c}{k}\left\|\eta_{1}-\eta_{2}\right\|_{k} \quad \forall \eta_{1}, \eta_{2} \in C([0, T] ; V) .
$$

So for $k>c$, the operator $\Lambda$ is a contraction on the space $C([0, T] ; V)$ endowed with the norm $\|\cdot\|_{k}$. Then by the Banach fixed point theorem, $\Lambda$ has a unique fixed point $\eta^{*} \in C([0, T] ; V)$, which concludes the proof.

Now, we have all the ingredients to prove Theorem 2.1.

Proof of Theorem 2.1. Existence. Let $\eta^{*} \in C([0, T] ; V)$ be the fixed point of $\Lambda$ and let $v_{\eta^{*}}$ and $u_{\eta^{*}}$ be the functions given by (3.5) and (3.6) for $\eta=\eta^{*}$. Let $\beta_{\eta^{*}}$ the solution of Problem $P_{\eta \beta}$ for $\eta=\eta^{*}$. We show that $\left(u_{\eta^{*}}, \beta_{\eta^{*}}\right)$ is a solution of Problem $P_{2}$. Indeed, choosing $\eta=\eta^{*}, g=g_{\eta^{*}}^{*}$ in (3.1) and using (3.5), we obtain

$$
\begin{array}{r}
\left\langle A \varepsilon\left(v_{\eta^{*}}(t)\right), \varepsilon(w)-\varepsilon\left(v_{\eta^{*}}(t)\right)\right\rangle_{Q}+\left(\eta^{*}(t), w-v_{\eta^{*}}(t)\right)_{V}+j\left(g_{\eta^{*}}^{*}(t), w\right) \\
\quad-j\left(g_{\eta^{*}}^{*}(t), v_{\eta^{*}}(t)\right) \geq\left(f(t), w-v_{\eta^{*}}(t)\right)_{V}, \quad \forall w \in V, t \in[0, T] .
\end{array}
$$

Let $\beta$ denote the solution of Problem $P_{\eta \beta}$ for $\eta=\eta^{*}$, i.e., $\beta=\beta_{\eta^{*}}$. As $\eta^{*}=$ $\Lambda \eta^{*}$, the inequality (2.20) follows from (3.4), (3.6) and (3.17), since $v_{\eta^{*}}=\dot{u}_{\eta^{*}}$ and $g_{\eta^{*}}^{*}=u_{\eta^{*}}$. The equality (2.22) follows from (3.6), and the regularity $u_{\eta^{*}} \in C^{1}([0, T] ; V)$ is a consequence of Lemma 3.1, (2.18) and (3.6). Clearly, equalities (2.21) and (2.23) hold by Problem $P_{\eta \beta}$. Also the regularity of the bonding field $\beta \in W^{1, \infty}\left(0, T ; L^{2}\left(\Gamma_{3}\right)\right) \cap \mathcal{O}$ follows from Lemma 3.3.

Uniqueness. Let $(u, \beta) \in C^{1}([0, T] ; V) \times W^{1, \infty}\left(0, T ; L^{2}\left(\Gamma_{3}\right)\right) \cap \mathcal{O}$ be a solution of Problem $P_{2}$ and denote by $\eta \in C([0, T] ; V)$ the function defined by

$$
(\eta(t), w)_{V}=\langle B \varepsilon(u(t)), \varepsilon(w)\rangle_{Q}+r(\beta(t), u(t), w) \forall w \in V, t \in[0, T],
$$

and let

$$
v=\dot{u} .
$$

Using (2.20) we infer that $v$ is a solution of the variational problem $P_{\eta u}$ and since this problem has a unique solution $v_{\eta u} \in C([0, T] ; V)$, we conclude that

$$
v=v_{\eta u} .
$$

Hence, from (2.22), (3.19) and (3.20) we obtain

$$
u(t)=u_{0}+\int_{0}^{t} v_{\eta u}(s) d s, \quad t \in[0, T],
$$

i.e., $u$ is a fixed point of $\Lambda_{\eta}$. It follows from Lemma 3.1 that $u=g_{\eta}^{*}$ and by (3.20) we have

$$
v=v_{\eta g_{\eta}^{*}} .
$$


Then (3.5) and (3.21) imply

$$
v=v_{\eta}
$$

So, it follows from $(2.22),(3.6),(3.19)$ and $(3.22)$ that

$$
u=u_{\eta} .
$$

Next, (2.21) and the initial condition $\beta(0)=\beta_{0}$ imply that $\beta$ is a solution of Problem $P_{\eta \beta}$, and since this problem admits a unique solution $\beta_{\eta}$, we conclude that

$$
\beta=\beta_{\eta}
$$

Using now (3.10), (3.18), (3.23), and (3.24) we deduce that $\Lambda \eta=\eta$, and as the operator $\Lambda$ admits a unique fixed point guaranteed by Lemma 3.3, it follows that

$$
\eta=\eta^{*}
$$

The uniqueness of the solution is now a consequence of (3.23)-(3.25).

\section{References}

[1] H. Brezis, Équations et inéquations non linéaires dans les espaces vectoriels en dualité, Ann. Inst. Fourier (Grenoble) 18 (1968), no. 1, 115-175.

[2] L. Cangémi, Frottement et adhérence: modèle, traitement numérique et application à l'interface fibre/matrice, Ph.D. Thesis, Univ. Méditerranée, Aix Marseille I, 1997.

[3] O. Chau, J. R. Fernández, M. Shillor and M. Sofonea, Variational and numerical analysis of a quasistatic viscoelastic contact problem with adhesion, J. Comput. Appl. Math. 159 (2003), 431-465.

[4] O. Chau, M. Shillor and M. Sofonea, Dynamic frictionless contact with adhesion, J. Appl. Math. Phys. 55 (2004), 32-47.

[5] M. Cocu and R. Rocca, Existence results for unilateral quasistatic contact problems with friction and adhesion, Math. Model. Numer. Anal. 34 (2000), 981-1001.

[6] G. Duvaut et J.-L. Lions, Les inéquations en mécanique et en physique, Dunod, Paris, 1972.

[7] J. R. Fernández, M. Shillor and M. Sofonea, Analysis and numerical simulations of a dynamic contact problem with adhesion, Math. Comput. Modelling 37 (2003), $1317-1333$.

[8] M. Frémond, Adhérence des solides, J. Mécanique Théor. Appl. 6 (1987), 383-407.

[9] —, Équilibre des structures qui adhèrent à leur support, C. R. Acad. Sci. Paris Sér. II 295 (1982), 913-916.

[10] - Non-Smooth Thermomechanics, Springer, Berlin, 2002.

[11] S. A. Nassar, T. Andrews, S. Kruk and M. Shillor, Modelling and simulations of a bonded rod, Math. Comput. Modelling 42 (2005), 553-572.

[12] M. Raous, L. Cangémi and M. Cocu, A consistent model coupling adhesion, friction, and unilateral contact, Comput. Meth. Appl. Mech. Engrg. 177 (1999), 383-399.

[13] R. Rochdi, M. Shillor and M. Sofonea, Quasistatic viscoelastic contact with normal compliance and friction, J. Elasticity 51 (1998), 105-126. 
[14] J. Rojek and J. J. Telega, Contact problems with friction, adhesion and wear in orthopeadic biomechanics. I: General developments, J. Theor. Appl. Mech. 39 (2001), 655-677.

[15] M. Shillor, M. Sofonea, and J. J. Telega, Models and Variational Analysis of Quasistatic Contact, Lecture Notes in Phys. 655, Springer, Berlin, 2004.

[16] M. Sofonea, W. Han, and M. Shillor, Analysis and Approximations of Contact Problems with Adhesion or Damage, Pure Appl. Math. 276, Chapman \& Hall/CRC Press, Boca Raton, FL, 2006.

[17] M. Sofonea, R. Arhab, and R. Tarraf, Analysis of electroelastic frictionless contact problems with adhesion, J. Appl. Math. 2006, art. ID 64217, 25 pp.

[18] M. Sofonea and T. V. Hoarau-Mantel, Elastic frictionless contact problems with adhesion, Adv. Math. Sci. Appl. 15 (2005), 49-68.

Arezki Touzaline

Laboratoire de Systèmes Dynamiques

Faculté de Mathématiques, USTHB

BP 32 El Alia

Bab-Ezzouar, 16111, Algeria

E-mail: ttouzaline@yahoo.fr

Received on 10.4.2009;

revised version on 30.9.2009 